\title{
Primary diffuse large B-cell lymphoma of the central nervous system: A case report and literature review
}

\author{
DAWEI CHEN ${ }^{1}$, WEIHONG GU ${ }^{2}$, WENZHONG LI ${ }^{1}$, XIAOLIANG LIU ${ }^{1}$ and XIAOYU YANG ${ }^{1}$ \\ ${ }^{1}$ Department of Neurosurgery, The First Hospital of Jilin University; ${ }^{2}$ Department of Surgery, \\ The Second Hospital of Jilin University, Changchun, Jilin 130021, P.R. China
}

Received February 4, 2015; Accepted December 14, 2015

DOI: $10.3892 / \mathrm{ol} .2016 .4365$

\begin{abstract}
The present study reports the clinical diagnosis and management of a patient with primary diffuse large B-cell lymphoma $(\mathrm{Bcl})$ of the central nervous system (CNS). Making an early diagnosis of primary diffuse large $\mathrm{Bcl}$ is challenging due to the variable and complicated clinical manifestations of the disease. The relevant literature was reviewed, and high-dose methotrexate, whole brain radiotherapy and highly active antiretroviral therapy was recommended for the patient. The present study elucidates the role of positron emission tomography-computed tomography scans for the diagnosis and management of primary diffuse large $\mathrm{Bcl}$ of the CNS, and demonstrates the importance of resection surgery in the management of the disease. Specifically, the present study proposes that resection surgery may be applied in the early stages of disease for patients with a single occupied lesion and increased intracranial pressure.
\end{abstract}

\section{Introduction}

Primary diffuse large B-cell lymphoma (Bcl) of the central nervous system (CNS) is a rare tumor of the brain and spinal cord, which has been estimated to account for $\leq 1 \%$ of all lymphomas, 4-6\% of all extranodal lymphomas and $\sim 1-3 \%$ of primary CNS tumors (1-3). Immunocompromised individuals are considered most at risk of the disease, however, the incidence of primary diffuse large Bcl of the CNS (PCNSL) is increasing in immunocompetent populations (4).

Due to the absence of a typical clinical presentation, multiple neuroimaging appearances, heterogeneity of the pathological morphology and no specific laboratory examination, the immunohistochemistry and molecular biology are of vital importance in accurate diagnosis of PCNSL (5). At present, the methods of treatment include surgery, chemotherapy

Correspondence to: Dr Dawei Chen, Department of Neurosurgery, The First Hospital of Jilin University, Building 3, 71 Xinmin Street, Changchun, Jilin 130021, P.R. China

E-mail: cdw001970@163.com

Key words: lymphoma, central nervous system tumor, diagnosis, treatment and radiotherapy (5). Although the introduction of systemic chemotherapy and radiotherapy has consistently improved survival, the prognosis of PCNSL remains poor (5), with a median survival time of 17-45 months following symptomatic treatment (6). The current study reports the case of a patient with PCNSL and reviews the literature regarding the presentation, diagnosis, treatment and prognosis of the disease. The present study was approved by the Ethics Committee of The First Hospital Of Jilin University (Changchun, China) and written informed consent was obtained from the patient.

\section{Case report}

A 58-year-old male presented to The First Hospital Of Jilin University (Changchun, China) with slurred speech and facial paralysis. A physical examination revealed that the left nasolabial fold was shallow. The patient denied a family history of genetic or immunodeficiency disorders. The cerebrospinal fluid (CSF) appeared to be normal and free from tumor cells. The ultrasound examination results and the peripheral blood tests, including for the human immunodeficiency virus and Epstein-Barr virus, were either normal or negative. The brain magnetic resonance imaging (MRI) scan revealed an occupied lesion of $1.8 \mathrm{~cm}$ at the right basal ganglia region and corona radiata (Fig. 1A). The patient refused a stereotactic biopsy; however, the patient's symptoms improved with symptomatic treatment. In particular, the patient's speech regained fluency, seven days subsequent to the administration of mannitol (50 g, three times daily) and dexamethasone (10 mg, twice daily) for three days.

Two weeks subsequent to the identification of the lesion, the patient received local $\gamma$ knife treatment at the Central Hospital of Changchun (Changchun, China) on September 23, 2012, and was re-admitted to the hospital on October 3, 2012, with a headache and numbness on the left side of the body that had lasted for 5 days. Another brain MRI scan revealed high and low signal in the right basal ganglia and radial area. The size of the lesion was $1.3 \times 1.2 \times 1.6 \mathrm{~cm}$. Compared with the previous MRI, the size of the lesion was significantly decreased (Fig. 1B). In addition, a single-voxel magnetic resonance spectroscopy (MRS) scan that used a point resolved spectroscopy protocol with a short $(35 \mathrm{msec})$ echo time revealed a notable elevation of the choline peak, a stable creatine peak and a decreasing $\mathrm{N}$-acetylaspartate (NAA) peak. 
A a

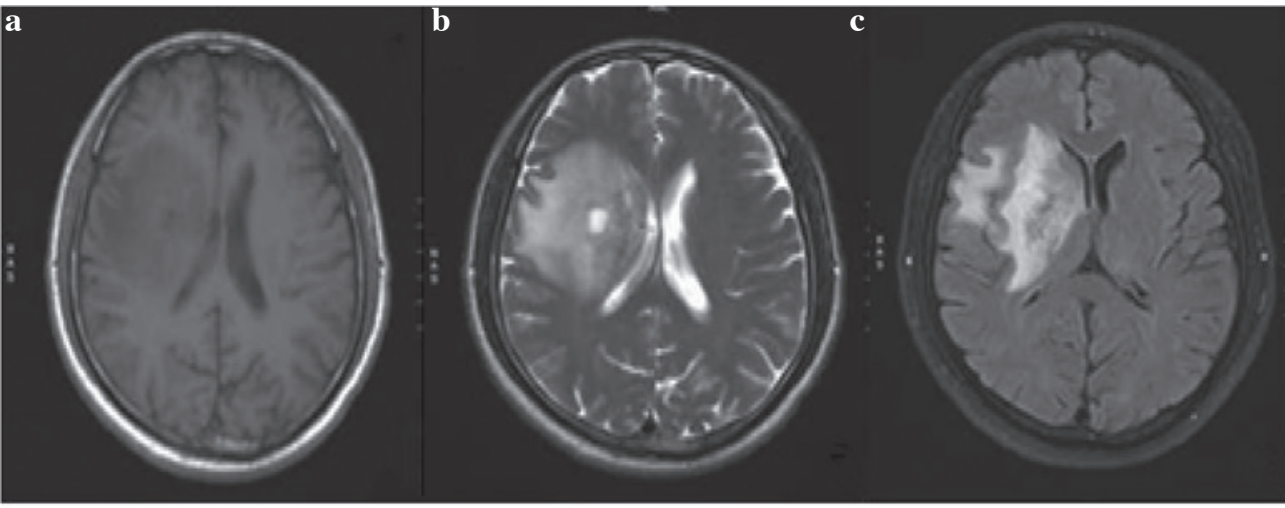

B

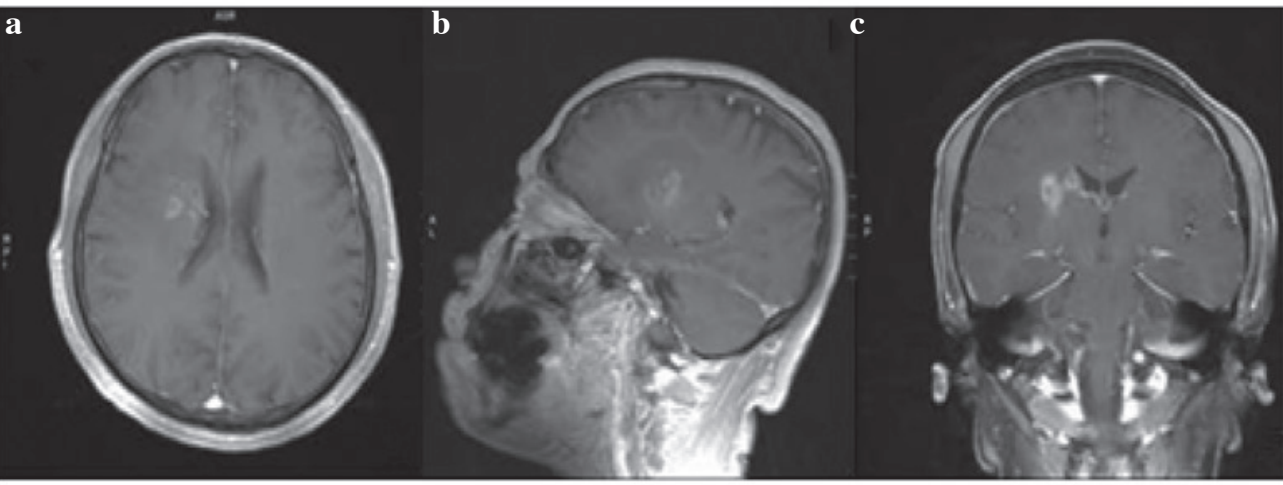

C $\mathbf{a}$

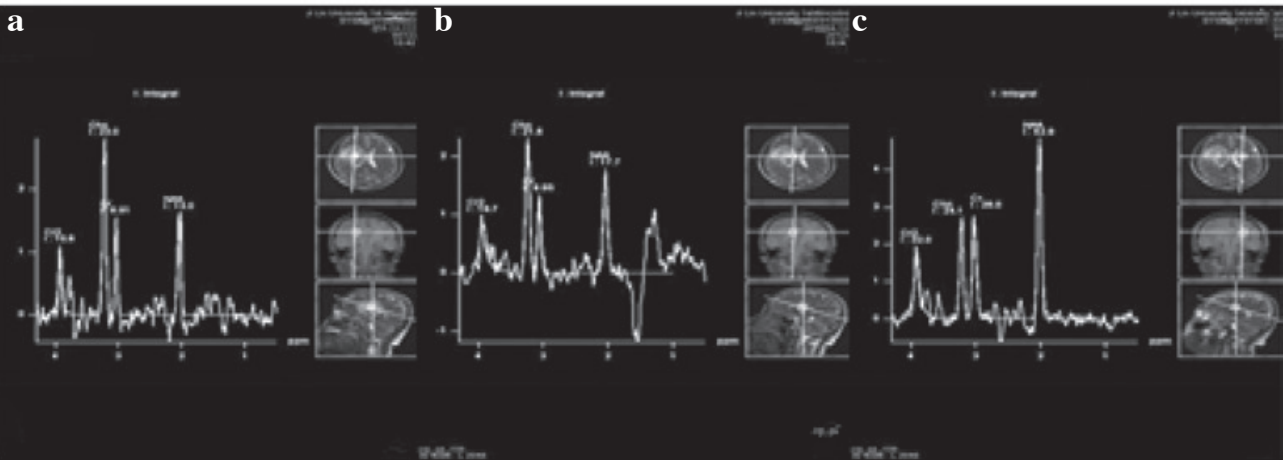

D

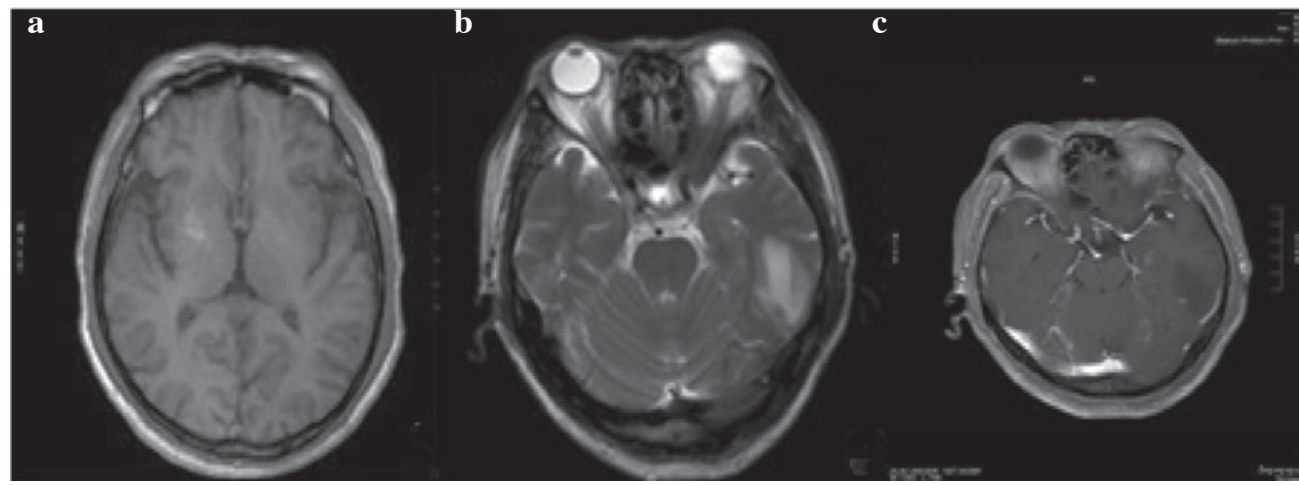

Figure 1. (A) Brain MRI scans identified an abnormal lesion on the right basal ganglia region and corona radiata. MRI images revealing (a) T1WI hypodensity, (b) T2WI hyperdensity and (c) fluid attenuation inversion recovery. (B) MRI scans showing a high and low signal in the (a) right basal ganglia and (b) radial area, and (c) a few patchy high signals in the central region, with low signal in the peripheral region. (C) Brain MRS revealing (a) a notable elevation of the choline peak and a stable creatine peak, and (b) a decreasing $\mathrm{N}$-acetylaspartate peak. MRS also revealed (c) a lactate peak and an elevation of the glutamate and glutamine peaks (2.1-2.5 ppm). The choline:creatine ratio was <3:1. (D) Brain MRI scans reveal (a) the lesion in the right basal ganglia and (b,c) that the radial area was barely visible. MRI, magnetic resonance imaging; MRS, magnetic resonance spectroscopy.

The MRS scan also revealed a lactate peak and elevation of the glutamate and glutamine peaks. These data indicated that the patient possessed a malignant tumor (Fig. 1C). One week later, 3 cycles of temozolomide therapy (first dose, $150 \mathrm{mg} / \mathrm{m}^{2}$; subsequent doses, $200 \mathrm{mg} / \mathrm{m}^{2}$ ) were administered.
Another brain MRI scan revealed that the size of the lesion was significantly decreased (Fig. 1D); however, the positron emission tomography-computed tomography (PET/CT) scan reported highly metabolic nodules in the right basal ganglia and left temporal lobe (Fig. 2A). Four months later, a follow-up 

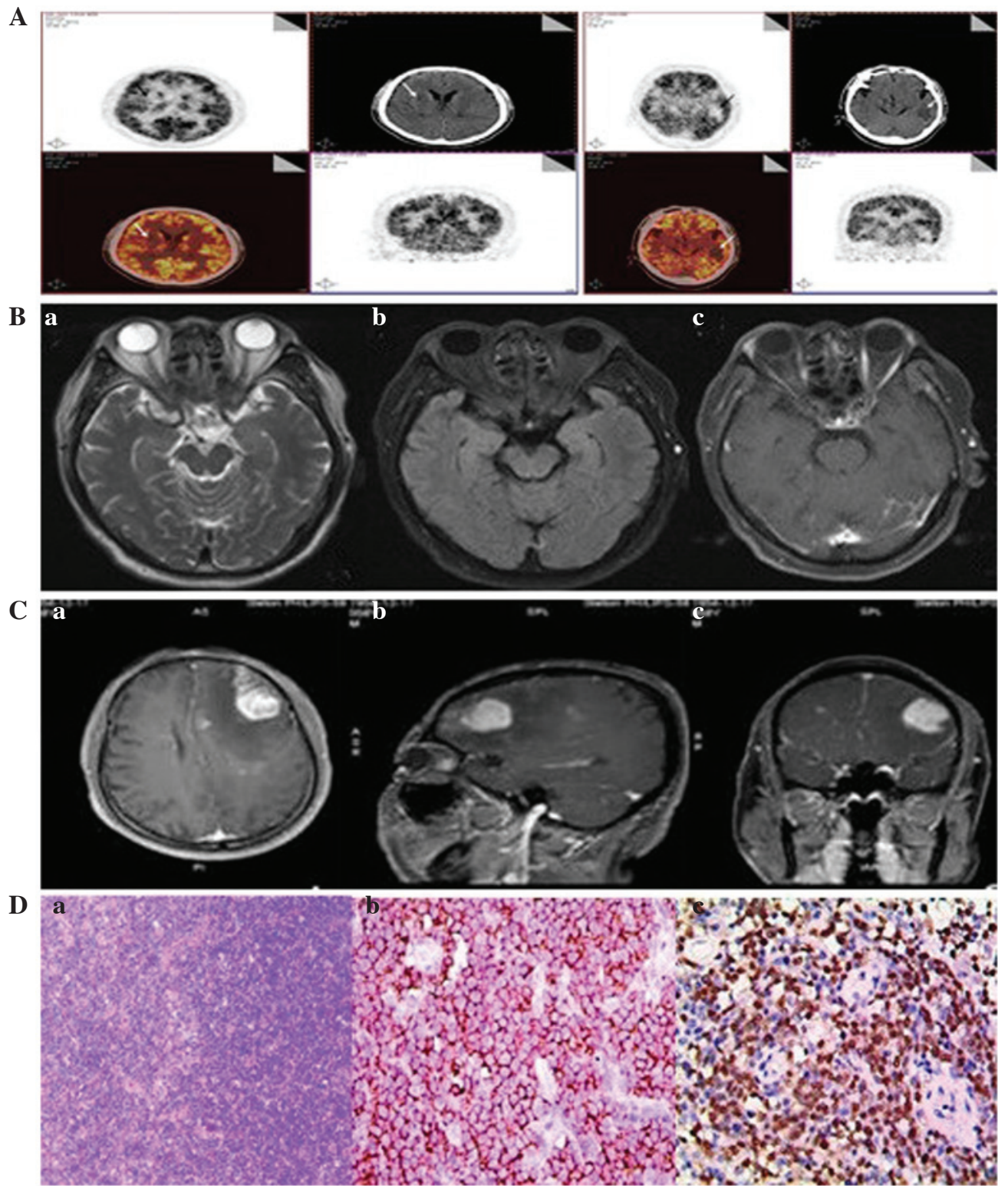

Figure 2. (A) PET/CT scans detected high metabolic nodules in the right basal ganglia and left temporal lobe (arrows). Images with a white background show PET and images with a black background and orange coloration show fusional PET/CT. (B) MRI scans revealed (a-c) that the left temporal lobe was hardly visible. (C) Brain MRI scans reveal (a) that the hyperdense lesion in the left frontal lobe was $5.5 \times 2.5 \times 1.8 \mathrm{~cm}$ in size, (b and c) that there was edema, and that the midline structure was shifted to the right. (D) Pathology revealed: (a) The tumor cell spreading; (b) CD20 cells in the tumor; and (c) multiple myeloma oncogene- $1^{+}$cells in the tumor. MRI, magnetic resonance imaging; PET-CT, positron emission tomography-computed tomography; CD20, cluster of differentiation 20 .

MRI indicated that the left temporal lobe lesion was resolved (Fig. 2B). Six months subsequent to the temozolomide treatment, the tumor appeared to be in full remission.

In July 2013, the patient was re-admitted to the hospital with weakness, slurred speech and memory loss that had lasted for 3 days. A neurological examination revealed receptive aphasia, weakness of the right side and shallowness of the right nasolabial fold. A brain MRI scan revealed a hyperdense lesion with a diameter of $5.5 \mathrm{~cm}$ in the left frontal lobe. Signs of edema and a rightward shift of the midline structure were also present (Fig. 2C). Additionally, there was an abnormal enhancement in the bilateral frontal lobe and right basal ganglia. A left frontal lobe tumor resection was performed. Intraoperatively, the tumor was gray red, soft and $4.8 \mathrm{~cm}$ in diameter. The tumor did not possess an envelope but had a rich blood supply. Postoperatively, the patient demonstrated notable improvements in speech and function, and exhibited no signs of complication.

A pathological examination of the resected tumor confirmed the diagnosis of PCNSL. The immunohistochemical analysis revealed that the tumor did not express the glial gibrillary acidic protein, cluster of differentiation (CD)3, CD43, cyclin D1, CD10, CD20, CD21, CD30, cancer antigen of $\mathrm{Ki}-67$, anaplastic lymphoma kinase or myeloperoxidase proteins (Fig. 2D). The tumor did express the paired box protein-5, Bcl-2, Bcl-6, B-cell related and forkhead box protein. In situ hybridization of the tumor identified Epstein-Barr virus-encoded RNA (Fig. 2D). The post-surgical MRI revealed the postoperative changes to the left frontal lobes without residual tumor (Fig. 3A). Another PET/CT scan 

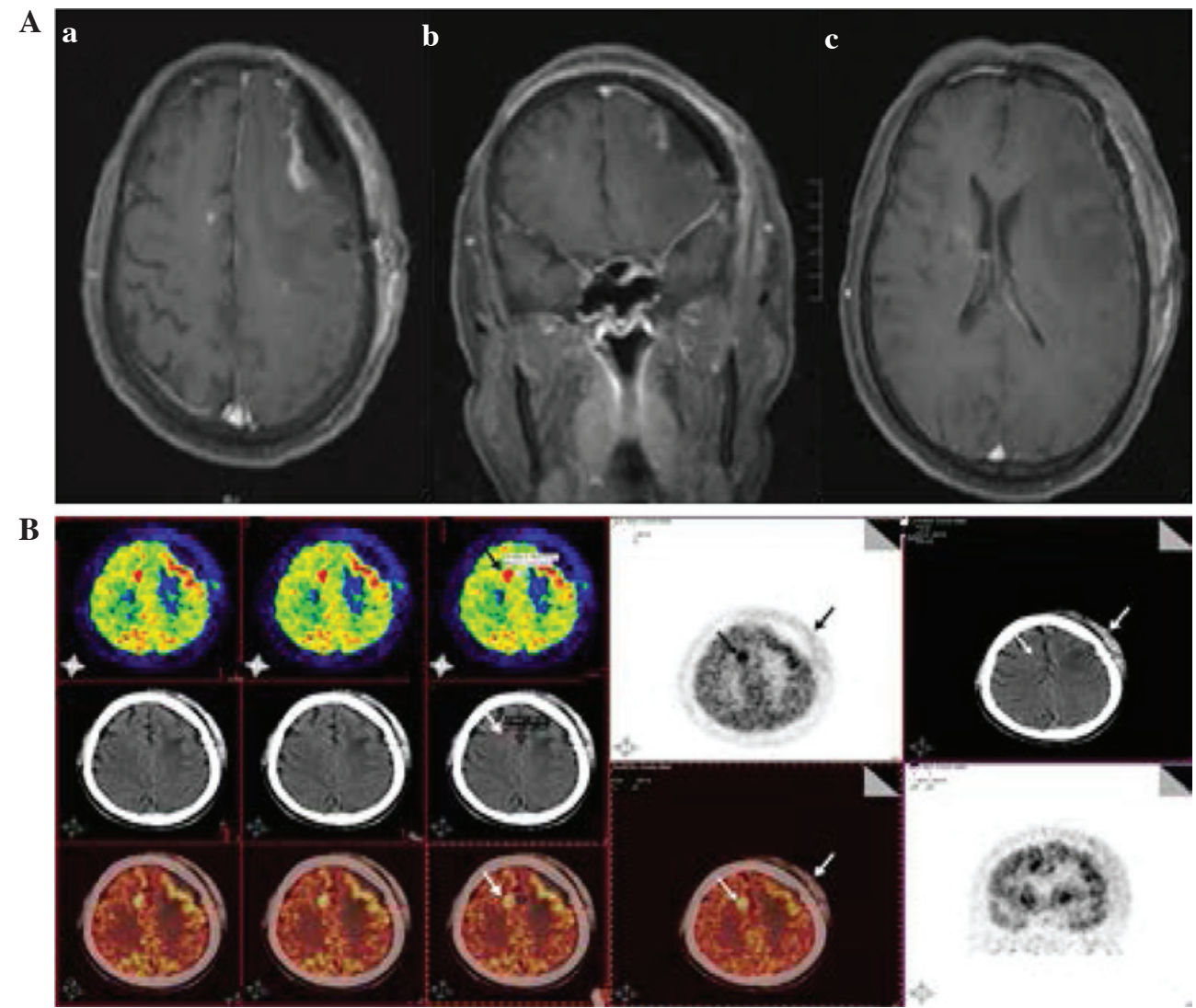

Figure 3. (A) Post-operative MRI scans revealed (a and b) the postoperative changes of the left frontal lobes and (c) that there was no residual tumor. (B) Postoperative PET/CT scans reveal high metabolic nodules in the right frontal lobe next to the falx cerebri with a standardized uptake value of 25. Images with a white background show PET, images with a black background and orange coloration show fusional PET/CT and images with a black background and green coloration show PET using a different color to highlight the nodule more clearly. MRI, magnetic resonance imaging; PET-CT, positron emission tomography-computed tomography.

revealed high metabolic nodules in the right frontal lobe next to the falx cerebri that demonstrated a maximum standardized uptake value (SUV) of 25 (Fig. 3B). In addition, the lactate dehydrogenase and macroglobulin levels and the sedimentation rate were all normal. The patient subsequently received six three-week cycles of high-dose methotrexate $\left(3.5 \mathrm{~g} / \mathrm{m}^{2}\right.$ daily; days 1,8 and 14) treatment, followed by whole-brain radiation therapy [40 Gy/25 fractions (f); once a week] for six weeks, and an additional dose of $9 \mathrm{~Gy} / 5 \mathrm{f} \mathrm{two} \mathrm{weeks} \mathrm{later.} \mathrm{The} \mathrm{patient}$ has demonstrated no signs of relapse and has remained stable during the 20-month follow-up.

\section{Discussion}

PCNSL is a B-cell non-Hodgkin's lymphoma that originates from, and localizes to, the cerebellum, spinal cord, pia mater, retina or optic nerve $(1,2)$. According to the updated WHO classification of tumors of haematopoietic and lymphoid tissues, the disease is recognized as a discrete entity (3). PCNSL has been characteristically associated with immunodeficient patients. However, the exact mechanism has not yet been identified (7). In immunocompetent populations, the median age of PCNSL occurrence is 53-57 years old, with a male:female ratio of 1.2:1. In immunocompromised populations, the median age of occurrence is 31-35 years old, with a male:female ratio of 7.38:1. However, the incidence of PCNSL in immunocompetent populations is increasing $(8,9)$.
PCNSL is characterized by a supratentorial localization. The clinical manifestations of PCNSL are similar to those of other intracranial tumors, including the high intracranial pressure and focal neurological deficit. The patient in the present study showed no evidence of immunodeficiency. The patient originally presented with dysarthria and received $\gamma$ knife and temozolomide treatment. The patient experienced 2 events of ectopic intracranial spreading.

The typical imaging characteristics of lymphoma may be used to differentiate between diagnoses. However, determining a diagnosis may be challenging; in particular, when attempting to rule out CNS metastasis, glioblastoma, inflammation, multiple sclerosis or other CNS demyelination. The most common imaging characteristics of PCNSL are hypointense $\mathrm{T} 1$ and hyperintense $\mathrm{T} 2$ signals, homogeneous or heterogeneous diffusion weighted imaging (DWI) and high circular signals. In addition, the gadopentetic acid enhancement may show a mass reinforcement, and the proton magnetic resonance spectroscopy may exhibit an elevation of choline and lactate peak. The fluorodeoxyglucose PET/CT scan may show single or multiple high metabolic signals that exhibit SUV elevation (10-18). In the present study, the MRI results differed from a typical PCNSL case: The lesion size was smaller and exhibited prominent surrounding edema, lateral ventricle compression and an evident occupied effect. Future diagnoses may be improved through the combined use of MRI scans with enhancement, DWI, 1-h MRS and PET/CT 
scans. However, pathological analysis remains to be the gold standard for the diagnosis of PCNSL.

Although the use of surgery for PCNSL patients has been debated $(19,20)$, the present study supports that surgical resections are critical for the management of PCNSL, particularly when used in combination with chemotherapy and radiotherapy regimens. Postoperatively, the symptoms of the patient in the present study have significantly improved without complication. The pathological analysis confirmed the diagnosis of PCNSL, and provided useful information for the additional management of the disease. Therefore, resection surgery is recommended to be the first treatment for patients with a single occupied lesion and increased intracranial pressure. However, MRI stereotactic biopsies may be used for patients with multiple sporadic lesions that are located deeply in the brain or any important functional area. Ideally, steroid therapy may not be commenced for 1-2 weeks following a biopsy (21). Lesions in multiple locations may be biopsied to avoid misdiagnosis.

Radiation and chemotherapy may markedly improve the survival rates of PCNSL patients, particularly when chemotherapy agents are administered prior to radiation therapy. Recently, high-dose methotrexate (HD-MTX) has been widely used for the treatment of PCNSL, of which intrathecal MTX delivers a systemic high dose of MTX $\left(8 \mathrm{~g} / \mathrm{m}^{2}\right)$ (22). Osmotic pressure drugs may also be applied to the open blood-brain barrier to facilitate drug delivery to the lesions (23).

A previous study demonstrated that five cycles of HD-MTX $\left(3.5 \mathrm{~g} / \mathrm{m}^{2}\right)$ and procarbazine $\left(100 \mathrm{mg} / \mathrm{m}^{2} /\right.$ day) is beneficial for patients with a $90 \%$ objective response rate and median survival of 60 months (20). Other combination treatments included high-dose MTX chemotherapy, temozolomide and rituximab $(24,25)$.

Temozolomide is a chemotherapy agent with a high oral bioavailability that may cross the blood-brain barrier and may be effective in patients with glioma, leukemia, melanoma and lymphoma. The National Comprehensive Cancer Network has recommended that temozolomide may be used for refractory PCNSL $(26,27)$. In regards to radiation therapy, whole brain irradiation of 40-50 Gy followed by localized irradiation of $60 \mathrm{~Gy}$ on regions of edema is recommended as the best regimen (22).

The patient initially developed a tumor in the left temporal lobe, which demonstrated ectopic dissemination following three courses of temozolomide treatment. The lesion was undetectable 6 months subsequent to the temozolomide therapy. This result indicates that temozolomide combined with radiotherapy may be highly effective for PCNSL patients. In addition, the patient received 6 cycles of high dose chemotherapy $\left(3.5 \mathrm{~g} / \mathrm{m}^{2} \mathrm{MTX}\right)$, whole brain radiation therapy (40 Gy/25 f) and additional radiotherapy of $9 \mathrm{~Gy} / 5 \mathrm{f}$, subsequent to pathological confirmation.

Previous studies have indicated that PCNSL patients only survive for 3-6 months without treatment $(12,28)$. Although comprehensive therapy improves progression-free and overall survival (OS) rates compared with untreated patients, the 5 -year survival rate of patients that receive treatment remains $20-25 \%$ (29). In addition, 35-60\% of patients experience ectopic recurrence within two years of diagnosis. Patients that experience the recurrence of PCNSL demonstrate an OS of
8-18 months. At present, there is no standard treatment for recurrent PCNSL (30). Subsequent to 20 months of follow-up, the patient in the present study had a normal life and experienced no additional recurrence.

Overall, clinicians are recommended to consider the following issues. The clinical and imaging findings of PCNSL are complicated and varied, particularly in the early phase of remission stages, due to unremarkable symptoms, non-typical imaging findings and the misdiagnosis as inflammation. Usually, the specificity and sensitivity of the detection rate of tumors in the CSF by cytological findings is low. Single-photon emission computed tomography and molecular biology technology have not been widely applied. A stereotactic biopsy may be a better method for the diagnosis of PCNSL. Steroid treatment may improve the clinical symptoms, but recurrence is common. For patients that are misdiagnosed with multiple sclerosis or sarcoma, glucocorticoid treatment may dissolve lymphoid cells and damage normal morphology. Therefore, an accurate diagnosis of PCNSL is critical to avoid complications from inappropriate treatment.

\section{References}

1. Gerstner ER and Batchelor TT: primary central nervous system lymphoma. Arch Neurol 67: 291-297, 2010.

2. Senocak E, Oguz KK, Ozgen B, et al: Parenchymal lymphoma of the brain on initial MR imaging: A comparative study between primary and secondary brain lymphoma. Eur J Radiol 79: 288-294, 2011.

3. Kluin PM, Deckert M and Ferry JA: Primary diffuse large B-cell lymphoma of the CNS. In: WHO Classification of Tumours of Haematopietic and Lymphoid Tissues. Swerdlow SH, Campo E, Harris NL, Jaffe ES, PileriSA,SteinH, Thiele J and Vardiman JW (eds). Vol 2. 4th edition. IARC Press, Lyon, pp240-241, 2008.

4. Sierra del Rio M, Rousseau A, Soussain C, Ricard D and Hoang-Xuan K: Primary CNS lymphoma in immunocompetent patients. Oncologist 14: 526-539, 2009.

5. Li Y, Liu F, Liu Y and Zhang J: The diagnosis and treatment of primary central nervous system lymphoma. Zhonghua Xue Ye Xue Za Zhi 35: 771-773, 2014 (In Chinese).

6. Aki H, Uzunaslan D, Saygin C, Batur S, Tuzuner N, Kafadar A, Ongoren $\mathrm{S}$ and $\mathrm{Oz} \mathrm{B}$ : Primary central nervous system lymphoma in immunocompetent individuals: A single center experience. Int J Clin Exp Pathol 6: 1068-1075, 2013.

7. Ferreri AJ and Marturano E: primary CNS lymphoma. Best Pract Res Clin Haematol 25: 119-130, 2012.

8. Soussain C and Hoang-Xuan K: primary central nervous system lymphoma: An update. Curr Opin Oncol 21: 550-558, 2009.

9. Mrugala MM, Rubenstein JL, Ponzoni M and Batchelor TT: Insights into the biology of primary central nervous system lymphoma. Curr Oncol Rep 11: 73-80, 2009.

10. Haque $S$, Law M, Abrey LE and Young RJ: Imaging of lymphoma of the central nervous system, spine, and orbit. Radiol Clin North Am 46: 339-361, 2008.

11. Server A, Josefsen R, Kulle B, Maehlen J, Schellhorn T, Gadmar $\varnothing$, Kumar T, Haakonsen M, Langberg CW and Nakstad PH: Proton magnetic resonance spectroscopy in the distinction of high-grade cerebral gliomas from single metastatic brain tumors. Acta Radiol 51: 316-325, 2010.

12. Barajas RF Jr, Rubenstein JL, Chang JS, Hwang J and Cha S: Diffusion-weighted MR imaging derived apparent diffusion coefficient is predictive of clinical outcome in primary central nervous system lymphoma. AJNR Am J Neuroradiol 31: 60-66, 2010.

13. Mohile NA, Deangelis LM and Abrey LE: The utility of body FDG PET in staging primary central nervous system lymphoma. Neuro Oncol 10: 223-228, 2008.

14. Karantanis D, O'eill BP, Subramaniam RM, Witte RJ, Mullan BP, Nathan MA, Lowe VJ, Peller PJ and Wiseman GA: 18F-FDG PET/CT in primary central nervous system lymphoma in HIV-negative patients. Nucl Med Commun 28: 834-841, 2007.

15. Kawai N, Okubo S, Miyake K, Maeda Y, Yamamoto Y, Nishiyama Y and Tamiya T: Use of PET in the diagnosis of primary CNS lymphoma in patients with atypical MR findings. Ann Nucl Med 24: 335-343, 2010. 
16. Raoux D, Duband S, Forest F, Trombert B, Chambonnière ML, Dumollard JM, Khaddage A, Gentil-Perret A, and Péoc'h M: primary central nervous system lymphoma: Immunohistochemical profile and prognostic significance. Neuropathology 30: 232-240, 2010.

17. Kinoshita M,Hashimoto N,Izumoto S, Okita Y,Kagawa N, Maruno $\mathrm{M}$, Ohnishi T, Arita N and Yoshimine T: Immunohistological profiling by B-cell differentiation status of primary central nervous system lymphoma treated by high-dose methotrexate chemotherapy. J Neurooncol 99: 95-101, 2010.

18. Kleihues P and Cavenee WK (eds): Pathology and Genetics of Tumours of the Nervous System. IARC Press, Lyon, pp198-203, 2000.

19. Angelov L, Doolittle ND, Kraemer DF, Siegal T, Barnett GH, Peereboom DM, Stevens G, McGregor J, Jahnke K, Lacy CA, et al: Blood-brain barrier disruption and intra-arterial methotrexate-based therapy for newly diagnosed primary CNS lymphoma: A multi-institutional experience. J Clin Oncol 27: 3503-3509, 2009.

20. Zhang D, Hu LB, Henning TD, Ravarani EM, Zou LG, Feng XY, Wang WX and Wen L: MRI findings of primary CNS lymphoma in 26 immunocompetent patients. Korean J Radiol 11: 269-277, 2010

21. Basso U and Brandes AA: Diagnostic advances and new trends for the treatment of primary central nervous system lymphoma. Eur J Cancer 38: 1298-1312, 2002.

22. Yang Y, He J, Dang Y, Xia X, Dai Y and Xu R: One case report and literature review of primary central nervous system non Hodgkin's lymphoma. Lin Chuang Shen Jing Wai Ke Za Zhi 5: 373-375, 2014 (In Chinese).

23. Abrey LE, Yahalom J and DeAngelis LM: Treatment for primary CNS lymphoma: The next step. J Clin Oncol 18: 3144-3150, 2000.
24. Glantz MJ, Cole BF, Recht L, Akerley W, Mills P, Saris S, Hochberg F, Calabresi P and Egorin MJ: High-dose intravenous methotrexate for patients with nonleukemic leptomeningeal cancer: Is intrathecal chemotherapy necessary? J Clin Oncol 16: 1561-1567, 1998.

25. Guha-Thakurta N, Damek D, Pollack C and Hochberg FH Intravenous methotrexate as initial treatment for primary central nervous system lymphoma: Response to therapy and quality of life of patients. J Neurooncol 43: 259-268, 1999.

26. Murakami M, Fujimaki T, Asano S, Nakaguchi H, Yamada SM, Hoya K, Yamazaki K, Ishida Y and Matsuno A: Combination therapy with rituximab and temozolomide for recurrent and refractory primary central nervous system lymphoma. Yonsei Med J 52: 1031-1034, 2011

27. Makino K, Nakamura H, Hide T and Kuratsu J: Salvage treatment with temozolomide in refractory or relapsed primary central nervous system lymphoma and assessment of the MGMT status. J Neurooncol 106: 155-160, 2012.

28. Yamanaka R, Morii K, Shinbo Y, Homma J, Sano M, Tsuchiya N, Yajima N, Tamura T, Hondoh H, Takahashi $\mathrm{H}$, et al: Results of treatment of 112 cases of primary CNS lymphoma. Jpn J Clin Oncol 38: 373-380, 2008.

29. Weaver JD, Vinters HV, Koretz B, Xiong Z, Mischel P and Kado D: Lymphomatosis cerebri presenting as rapidly progressive dementia. Neurologist 13: 150-153, 2007.

30. Suh C, Kim JE and Yoon DH: Relapse pattern and prognostic factors for patients with primary CNS lymphoma. Korean J Hematol 47: 155-156, 2012. 